\title{
Comparison of Pixel and Subpixel Retinal Vessel Tree Segmentation Using a Deformable Contour Model
}

\author{
L. Espona ${ }^{1}$, M.J. Carreira ${ }^{1}$, M.G. Penedo ${ }^{2}$, and M. Ortega ${ }^{2}$ \\ ${ }^{1}$ Computer Vision Group. Electronics and Computer Science Dpt. University of Santiago de \\ Compostela. Spain \\ luciaep@usc.es, mjose@dec.usc.es \\ 2 VARPA Group. Computing Dpt. University of A Coruña. Spain \\ mgpenedo, mortega@udc.es
}

\begin{abstract}
This paper presents a comparison of pixel and subpixel performance of the snake-based system designed to detect the vessel tree in eye fundus images. The automatic analysis of the retinal vessel tree facilitates the computation of the arteriovenous index, which is essential for the diagnosis and evolution of several eye diseases. A high accuracy is required to correctly assess the clinicians and it is insufficient when working at a pixel level. The developed model is inspired in the classical snake but incorporating domain specific knowledge and profits from the automatic localization of the optic disc and from the extraction of vascular tree centerlines previously developed in our research group [1]. The efficiency and accuracy of the detection of arteriovenous structures are evaluated using the publicly available DRIVE database and an equivalent system configuration for pixel and subpixel results. Results demonstrate that, although more time consuming, subpixel retinal vessel extraction is much more reliable, keeping relatively low values of computing time.
\end{abstract}

Keywords: snakes, segmentation, retinal vessel tree, eye fundus image, subpixel.

\section{Introduction}

The automatic analysis of blood vessels is becoming more and more important in many clinical investigations and scientific researches related to vascular features. The early diagnosis of several pathologies, such as arterial hypertension, arteriosclerosis or diabetic retinophaty could be achieved analysing the vascular structures. The Digital Colour Fundus Photographs here used are a non invasive and innocuous technique to obtain the retinal vascular tree. Moreover, a specific CAD system is also necessary in large-scale ocular screening programs to make the ophthalmologist diagnosis process more efficient and accurate [2]. The retina arteriovenous index (AV index) indicates the relation between afferent and efferent blood vessels, that is arteries and veins of the retina. This index takes a vital priority in order to diagnose these illnesses and evaluate their consequences [3].

This paper deals with the selection of an appropiate precision level for our vascular tree detection system, evaluating its performance at pixel and subpixel level. This segmentation of the vascular tree would constitute the first step to allow the precise and robust AV index measuring.

The eye fundus images are quite problematic 2-D medical images. The main difficulties in them are the inadequate contrast, lighting variations and remarkable noise 
influence mainly due to its acquisition. Another drawback is the anatomic variability depending on the particular patient, affecting both the retinal background texture and the blood vessels structure. Blood vessels particular features make them complex structures to detect as the color of vascular structures is not constant even along the same vessel. Their tree-like geometry is often strange and complicated, including bifurcations and overlaps that may mix up the detection system. Nevertheless, other characteristics like the linearity or the tubular shape, make the vessel detection easier.

As blood vessels segmentation becomes essential for several medical diagnostic systems, numerous research efforts have been done in this field [4]. The vascular detection has been tackled from different approaches and techniques including pattern recognition, pixel-based approaches [5] or classification methods [6]. The deformable contour models are widely followed in vessel tracking, even combined with other techniques [7].

Our innovative system incorporates domain specific knowledge into the generic contour deformable model. The snake model is specialised with the blood vessels topological properties, which determine the detection system behaviour. We have taken a great advantage of the vascular tree graph, composed by the vessels centerlines obtained from a creases extraction system previously developed by our research group [1].

The decision between pixel or subpixel precision is very important for the arteriovenous index calculus, as a compromise between accuracy and efficiency measurement of vessel diameters is needed. Other approaches for vessel segmentation also work at a subpixel level, such as a fuzzy-model based segmentation method [8] or the ridge based vessel segmentation in color images of the retina [9].

In our system initialization, the original image is converted to grayscale and resampled with bi-cubic interpolation to work at a subpixel level. This increases the computational cost because of the re-sampling time and the model complexity. Thus, the pixel level performance is more efficient but the precision achieved is not great enough (especially in terms of sensitivity), as we will show in the results section. In the evaluation we consider mainly the execution times, the accuracy, the sensitivity and the specificity of the segmentation process.

Next we will explain briefly our vessel tree detection system, beginning with crease extraction in order to perform the deformable contour evolution. A more detailed description of our model can be found in [10].

\section{Vessel Tree Detection System}

The developed model for the detection of the vessel tree is based on a deformable contour guided by vessel creases. This section will begin explaining the creases extraction process, then the classical deformable contour model will be described. Once the theorical fundamentals have been presented, our particular snake model will be analysed in depth, presenting the innovative specific features and their resulting behaviour.

\subsection{Creases Extraction}

A crease is a continuous area of points on the image, shaping a highest or a lowest level in its environment. In this way, blood vessels can be considered as regions which 
form an extreme and tubular level on their neighbourhood. This fact allows to locate the vessels by using the creases position (see Fig 1 a)). The creases extraction is essential for the detection process, since it will determine the initial snake and will act as external energy guiding the contour expansion. The creases extraction [1] is a crucial and irreversible step in the snake evolution. If a crease is not detected, the corresponding vessel will remain unsegmented. Thus, we have enhanced the creases image by exploiting an algorithm developed by our research group, part of a biometric authentication system [11]. The feature points (ridge endings and bifurcations) obtained with this system are adequately connected to get rid of disconinuites along the centerline (see Fig@(b)). These ridge lines will guide the snake in their growing, as we will show in next subsection.

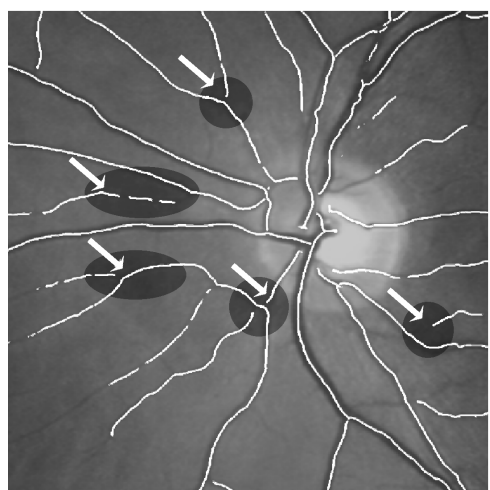

(a)

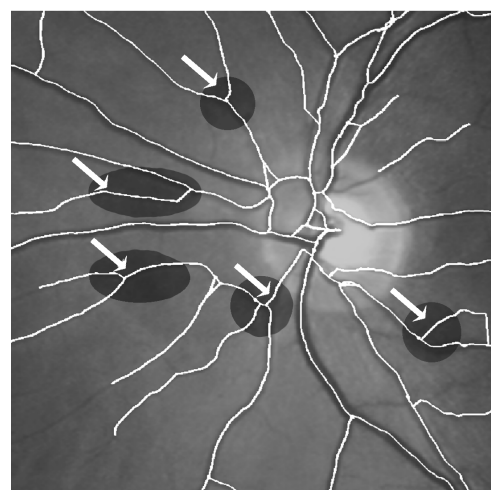

(b)

Fig. 1. Creases image: (a) A retinal image with its creases overlapped in white, corresponding to the vessel centerlines. (b) Creases image enhanced in terms of continuity.

\subsection{Deformable Contour Model}

Our approach is based on the deformable contour model, also called snake model, proposed by Kass et al. [12] to segment objects in 2-D images.

This particular snake model will not consider the classical internal energy as the vessel shape may be very tortuous. Thus, the global energy affecting the snake will be defined as a set of five energies and weighting factors [10]:

$$
\varepsilon=\varepsilon_{\text {ext }}+\varepsilon_{\text {int }}=\gamma \varepsilon_{\text {edge }}+\delta \varepsilon_{\text {cres }}+v \varepsilon_{\text {dir }}+\sigma \varepsilon_{\text {mark }}+\omega \varepsilon_{\text {dif }}
$$

The terms $\varepsilon_{e d g e}, \varepsilon_{\text {cres }}$ and $\varepsilon_{d i r}$ correspond to the external energy, whereas $\varepsilon_{\text {mark }}$ and $\varepsilon_{\text {dif }}$ represent the internal energy. The first term $\varepsilon_{\text {edge }}$ corresponds to the edge distance energy that helps the snake advance of nodes close to vessel boundaries but it also stops them when they reach a minimum, that is, when they reach an edge point. The second term $\varepsilon_{\text {cres }}$ corresponds to the creases distance energy that drives the snake along the arteriovenous structure and blocks it if a maximum distance threshold is reached. The inflate pressure $\varepsilon_{d i r}$ is the strongest expansion force of the snake. Each node has one 
assigned direction that determines the three adjacent possible positions to avoid that a node can go through an edge and go on moving out of the vessel boundary. The fourth term $\varepsilon_{\text {mark }}$ is the marker energy that constraints the snake dilation as nodes are moving to ensure that self overlapping or turning back never happens. The difference energy $\varepsilon_{\text {dif }}$ reinforces the precision of the snake expansion as it hints the nodes to occupy positions different from its neighbours situations.

Based on the external energy described in equation (1), three possible node states are defined : normal, crease and edge. The nodes in the crease state are located in the vessel crease and they make the snake to advance along the vessel centerline. The positions close to vessel boundaries are occupied by nodes in the edge state that tend to become stable when reaching the vessel edge. The rest of nodes are in the normal state and they contribute to the snake expansion in an intermediate direction.

To define the contour evolution, first we have to deal with the snake initialisation. Firstly, the creases and edges images and energy maps are calculated on the original image (resampled when using subpixel segmentation). Next, a circumference surrounding the optical nerve is traced, using the automatic localization system developed by our research group [13]. The intersections of this circumference with creases will be the seed nodes of the snake.

After its initialisation, the snake evolves following a deterministic and iterative algorithm to minimise these energy functions locally until the stability is reached. Each active node tries to move towards a lower energy position until it becomes irreversibly inactive when arriving to an edge or due to the control operations [10]. To completely segment the vascular structure, the snake grows by new active nodes insertion considering an euclidean distance threshold.

At a given moment, the snake nodes have a position in the image space and an assigned node state: edge, normal or crease. The energy for each possible node movement is calculated considering the energy terms values associated to the position and the weights associated to the node. Iteratively, each vertex is moved according to forces that work on it, that is towards the local minimum energy situation. Consequently, the whole contour expands and the snake flows inside the vessel covering the vascular branch. In addition, we perform control operations considering vessel structural features to avoid problems derived from edge discontinuities [10].

\section{Results}

In this section we report the results of vessel segmentation obtained by our snake model on medical images from the publicly available DRIVE database [9]. The test set here used contains 20 JPEG compressed images acquired using a Canon CR5 non-mydriatic $3 \mathrm{CCD}$ camera with a 45 degree field of view (FOV). Originally, the images were of size $768 \times 584$ and 8 bits per color plane but we converted them into grayscale images (256 gray levels). The manual segmentation results and the FOV mask images for computing the performance measures were provided together with the DRIVE database.

A similar configuration has been empirically set for all pixel and subpixel results mainly by doubling the distance parameters. In the initialisation, equivalent centre and 
Table 1. Average performance of our pixel and subpixel snake models and an independent observer $\left(2^{\text {nd }} \mathrm{Ob}\right.$.), with the same human segmentation as ground truth

\begin{tabular}{cccc} 
& Accuracy & Sensitivity & Specifity \\
\hline Pixel & $\mathbf{0 . 9 2 2 3}$ & 0.6615 & 0.9575 \\
Subpixel & $\mathbf{0 . 9 3 2 5}$ & 0.7313 & 0.9600 \\
$2^{\text {nd }}$ Ob. & $\mathbf{0 . 9 4 8 9}$ & 0.7676 & 0.9736
\end{tabular}

radius to get the intersection points have been defined on each image. The system performance measures (see Table 1) consider only the FOV region without the optic disc.

Both systems performance results are satisfying in terms of accuracy and specificity, using the manual segmentation of the first observer as ground truth. The subpixel system achieves a more sensitive segmentation than the pixel model. The sensitivity values are not so high even at the subpixel level, because of very thin vessels, but these thin vessels are not particularly relevant for the AV index estimation.

Regarding to the efficiency, the model has been implemented in $\mathrm{C}++$ and executed on a PC with a T2400 Core Duo processor $(1.83 \mathrm{GHz})$ and $2 \mathrm{~Gb}$ memory. The optic disc automatic detection has been excluded from time costs because it is still being improved and optimized. The bicubic resampling performed in the subpixel system has been optimized ignoring the black areas of the image that lay outside the FOV. The time measurements for each execution step of pixel and supixel segmentation are shown in Table 2

Table 2. Average execution time comparison in seconds for the proposed vessel detection model working at pixel and subpixel levels. $\mathrm{T}_{\text {res }}$ corresponds to double size resampling. The extraction is $\mathrm{T}_{c r}$ for creases and $\mathrm{T}_{e d}$ for edges. $\mathrm{T}_{c r E n}$ and $\mathrm{T}_{e d E n}$ correspond to the the energy images calculation for creases and edges, respectively. The snake evolution is represented by $\mathrm{T}_{\text {snk }} . \mathrm{T}_{\text {total }}$ is the whole image segmentation process time.

\begin{tabular}{cccccccc}
\multicolumn{10}{c}{ Time Costs } \\
Level & $\mathrm{T}_{r e s}$ & $\mathrm{~T}_{c r}$ & $\mathrm{~T}_{c r E n}$ & $\mathrm{~T}_{e d}$ & $\mathrm{~T}_{e d E n}$ & $\mathrm{~T}_{\text {snk }}$ & $\mathrm{T}_{t o t}$ \\
\hline Pixel & 0 & 2.0 & 0.2 & 1.4 & 0.2 & 0.6 & $\mathbf{4 . 4}$ \\
Subpixel & 4.7 & 10.0 & 0.4 & 11.7 & 1.2 & 3.7 & $\mathbf{3 1 . 7}$
\end{tabular}

For each image, the average time needed for the whole vessel detection process at a pixel level $\mathrm{T}_{\text {total }}$ is 4.4 seconds (see Table 2). When working at a subpixel level, this time is higher (about 31.7 seconds). The pixel segmentation doesn't need a resampled image, so the 4.7 seconds corresponding to Tres are saved.

Other important steps are extracting the creases $\left(\mathrm{T}_{c r}\right)$ and obtaining the edges $\left(\mathrm{T}_{e d}\right)$ as they roughly represent the $70 \%$ of the whole process. The creases extraction and the edge calculation are notably slower at a subpixel level. In addition to the doubled image size, this difference is caused by the configuration of the creases extractor and the edge filter [14]. As the interpolation sligthly smooths the image, a higher sensitivity is required and thus the image processing efficiency is affected.

Calculating the energy images $\left(\mathrm{T}_{c r E n}\right.$ and $\left.\mathrm{T}_{e d E n}\right)$ takes a time directly proportional to the influence distance selected, therefore it is longer for subpixel segmentation as a 
higher limit is selected. Anyway, energy images calculation is not a high time-consuming step. The snake evolution itself $\left(\mathrm{T}_{\text {snk }}\right)$ takes less than a second for pixel segmentation and 3.7 seconds at a subpixel level. This step is completed after an average of 932 iterations at a pixel level, but the subpixel segmentation needs approximately the double ( 1916 iterations). The snake size has a significant incidence on this step duration, as it affects the number of nodes, hence the calculations per iteration.

\section{Conclusions and Discussion}

In conclusion, our snake-based methodology to segment the vessel tree on retinal digital images requires to work at a subpixel level to obtain precise results. What the subpixel segmentation slightly lacks in efficiency, it makes up with the increase of accuracy achieved.

The comparison between pixel and subpixel segmented images (see Fig.2) makes clear the improvement of subpixel precision. A lot of thin vessels are unsegmented, therefore the pixel sensitivity is very low. Even the pixel segmentation of wide vessels is

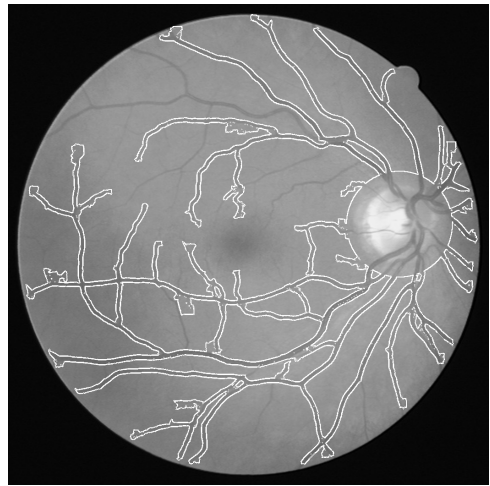

(a)

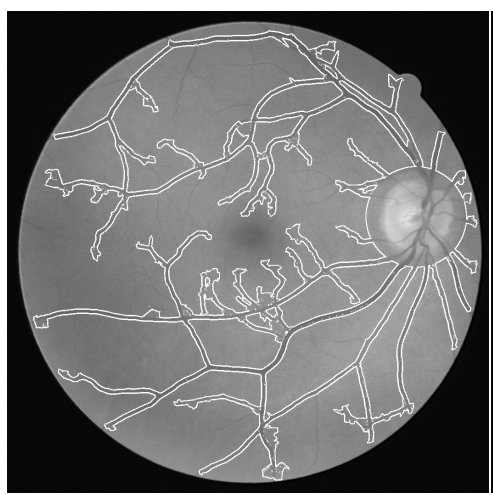

(c)

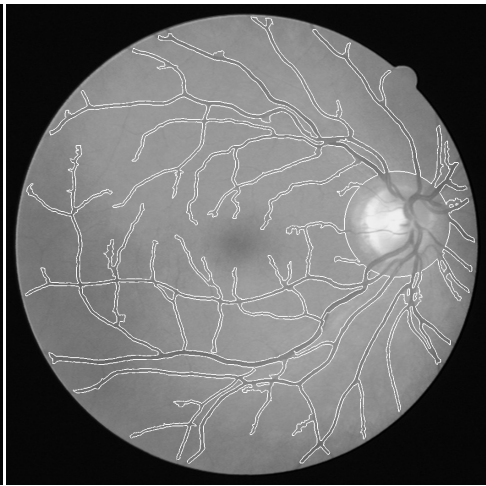

(b)

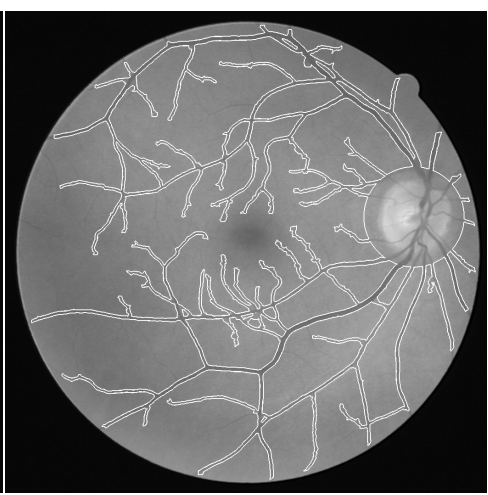

(d)

Fig. 2. (a) and (c):Pixel vessel detection results. (b) and (d) Subpixel vessel detection results. 
not very precise in comparison with the subpixel system (see Table 1). On the contrary, the subpixel segmentation accuracy is similar to human performance, although it is not able to segment some very thin vessels. Actually, very thin vessels are not esential for the AV index calculation, since the a ophthalmologists are only interested on main vessels leading to reproducible results. The pixel level results could be useful for certain applications, where the efficiency is critical and the accuracy requirements are low. A precise segmentation such as the one obtained working at a subpixel level is suitable for a wide range of utilities related to retinal or vascular pathologies. To set an example, removing the vessel tree detected could make easier the location of retinal background lesions.

Table 3. Results for different segmentation methods and an independent observer $\left(2^{\text {nd }} \mathrm{Ob}\right.$.), with the same human segmentation as ground truth. The proposed snake-based method results are evaluated excluding (Subpixel) and including the segmentation of the optic disc area (Subpixel (including O.D.)).

\begin{tabular}{|c|c|c|c|c|c|}
\hline & Accuracy & Sensitivity & Specifity & ROC Area & Time (s) \\
\hline Subpixel & 0.9325 & 0.7313 & 0.9600 & & 31.7 \\
\hline Subpixel (including O.D.) & 0.9300 & 0.7272 & 0.9598 & & 31.9 \\
\hline $2^{\text {nd }} \mathrm{Ob}$ & 0.9473 & 0.7760 & 0.9725 & & 7200.0 \\
\hline Soares et al. [6] & 0.9466 & & & 0.9614 & 180.0 \\
\hline Mendoça [5] & 0.9463 & 0.7315 & 0.9781 & & 150.0 \\
\hline Staal et al. [9] & 0.9441 & 0.719 & 0.977 & 0.9520 & 900.0 \\
\hline
\end{tabular}

A extreme efficiency in terms of execution time cost has been achieved for pixel segmentation. The subpixel segmentation needs more time but it is still a short process compared with the tedious and long manual detection (about two hours each image). Other state-of-the-art segmentation methods have much higher time costs, although they obtain similar accuracy values (see Table 3). Soares et al. [6] achieved an accuracy of 0.9466 but spending more than 3 minutes for each image, appart from the training time. Mendoça et al. [5] results need about 2.5 minutes considering just the algorithm for an accuracy of 0.9463 and Staal et al. [9] ridge based method get an accuracy of 0.9441 in 15 minutes per image.

We developed a method to allow our system to segment the whole image in order to exactly compare the performance results. Another snake is created from the same initial contour but it evolves inversely, segmenting the optic disc. The performance results are slightly lower, because the model is not optimised for this inverse segmentation. The subpixel system reaches an average accuracy of 0.9300 in less than 32 seconds, because it does not need to perform any complicated image preprocessing and it only handles one snake instance for the whole vascular tree. This execution time per image is small enough for large screening and real-time applications. The reported performance results at a subpixel level are very encouraging, as the remarkable accuracy and specificity shows. The unsegmentation of the very thin vessels could be partially solved by a dynamical tuning of the vessel width parameter.

Our researching efforts are now mainly focused on automatically tuning the parameters depending on the image and on enhancing and optimising the energy minimisation. 
Acknowledgements. This paper has been partly funded by the Ministerio de Ciencia y Tecnología and the Xunta de Galicia through grant contracts TIC2003-04649-C02 and PGIDIT04PXIC20602PN. We also would like to thank the authors of the DRIVE database for making their data publicly available [9].

\section{References}

1. Caderno, I.G., Penedo, M.G., Mariño, C., Carreira, M.J., Gómez-Ulla, F., González, F.: Automatic Extraction of the Retina AV Index. In: Campilho, A.C., Kamel, M. (eds.) ICIAR 2004. LNCS, vol. 3212, pp. 132-140. Springer, Heidelberg (2004)

2. Niemeijer, M., van Ginneken, B., Staal, J., Suttorp-Schulten, M.S.A., Abràmoff, M.D.: Automatic Detection of Red Lesions in Digital Color Fundus Photographs. IEEE Transactions on Medical Imaging 24(5), 584-592 (2005)

3. Aurell, E.: A Note of Signs in the Fundus Oculi Hypertension Conventional Assessment and Significance. Bull. World Health Organ. 34, 955-960 (1967)

4. Niemeijer, M., Staal, J., van Ginneken, B., Loog, M., Abràmoff, M.D.: Comparative Study of Retinal Vessel Segmentation Methods on a new Publicy Avaliable Database. In: Proceedings of the SPIE. Medical Imaging 2004: Image Processing, vol. 5370, pp. 648-656 (2004)

5. Mendoça, A.M., Campilho, A.: Segmentation of Retinal Blood Vessels by Combining the Detection of Centerlines and Morphological Reconstruction. IEEE Transactions on Medical Imaging 25(9), 1200-1213 (2006)

6. Soares, J.V.B., Leandro, J.J.G., Cesar Jr., R.M.C., Jelinek, H.F., Cree, M.J.: Retinal Vessel Segmentation Using the 2-D Gabor Wavelet and Supervised Classification. IEEE Transactions on Medical Imaging 25(9), 1214-1222 (2006)

7. Toledo, R., Orriols, X., Binefa, X., Redeva, P., Vitriá, J., Villanueva, J.J.: Tracking elongated structures using statistical snakes. In: Proceedings IEEE Conference on Computer Vision and Pattern Recognition, vol. 1(1), pp. 157-162 (2000)

8. Phaml, T.D., Tran, D.T., Brown, M., Lee Kennedy, R.: Image Segmentation of Retinal Vessels by Fuzzy Models. In: Proceedings of International Symposium on Intelligent Signal Processing and Communication Systems (2005)

9. Staal, J.J., Abràmoff, M.D., Niemeijer, M., Viergever, M.A., van Ginneken, B.: Ridge based vessel segmentation in color images of the retina. IEEE Transactions on Medical Imaging 23, 501-509 (2004)

10. Espona, L., Carreira, M.J., Ortega, M., Penedo, M.G.: A Snake for Retinal Vessel Segmentation. In: Martí, J., Benedí, J.M., Mendonça, A.M., Serrat, J. (eds.) IbPRIA 2007. LNCS, vol. 4478, pp. 178-185. Springer, Heidelberg (2007)

11. Ortega, M., Mariño, C., Penedo, M.G., Blanco, M., González, F.: Personal Authentication based on Feature Extraction and Optic Nerve Location in Digital Retinal Images. Wseas Transactions on Computers 5(6), 1169-1176 (2006)

12. Kass, M., Witkin, A., Terzopoulos, D.: Active Contour Models. International Journal of Computer Vision 1(2), 321-331 (1998)

13. Blanco, M., Penedo, M.G., Barreira, N., Penas, M., Carreira, M.J.: Localization and Extraction of the Optic Disc using the Fuzzy Circular Hough Transform. In: Rutkowski, L., Tadeusiewicz, R., Zadeh, L.A., Żurada, J.M. (eds.) ICAISC 2006. LNCS (LNAI), vol. 4029, pp. 713-721. Springer, Heidelberg (2006)

14. Canny, J.: A Computational Approach to Edge-Detection. IEEE Transactions on Pattern Analysis and Machine Inteligence 8(6), 679-689 (1986)

15. Niemeijer, van Ginneken, B.: Image Sciences Institute.DRIVE: Results Browser (2002), http://www.isi.uu.nl/Research/Databases/DRIVE/browser.php 